\title{
Health Monitoring and Diagnosis of Equipment Based on Multi-sensor Fusion
}

\author{
https://doi.org/10.3991/ijoe.v14i04.8315 \\ Xuemei Yao $\left.{ }^{\varpi}\right)$, Shaobo Li, Yong Yao, Xiaoting Xie \\ Guizhou University, Guiyang, China \\ yaomei0119@126.com
}

\begin{abstract}
As the information measured by a single sensor cannot reflect the real situation of mechanical devices completely, a multi-sensor data fusion based on evidence theory is introduced. Evidence theory has the advantage of dealing with uncertain information. However, it produces unreasonable conclusions when the evidence conflicts. An improved fusion method is proposed to solve this problem. Basic probability assignment of evidence is corrected according to evidence and sensor weights, and an optimal fusion algorithm is selected by comparing an introduced threshold and a conflict factor. The effectiveness and practicability of the algorithm are tested by simulating the monitoring and diagnosis of rolling bearings. The result shows that the method has better robustness.
\end{abstract}

Keywords-Multi-sensor fusion; Monitoring and diagnosis; Evidence theory; Robustness

\section{Introduction}

Manufacturing is the mainstay of the national economy and the foundation of a powerful nation. The healthy operation of mechanical equipment is the prerequisite for the development of the manufacturing. The failure of various equipment parts will lead to major accidents and bring great losses to the enterprises [1]. Based on mechanics and information theory, health monitoring adopts multidisciplinary fusion technology to judge whether the equipment has achieved the expected functions and performance. Its essence is pattern recognition [2]. There are mainly three methods: offline analysis, online diagnosis and remote detection [3]. The offline analysis collects field data by regular inspection, then stores it to computer and diagnoses it by software. This method is incapable of sudden failure. Online analysis, by means of sensors and data acquisition module, detects the current state of the device, captures unexpected faults and analyzes it in time, so it is the most widely used in industry. Remote detection gives full play to the advantages of information exchange and resource sharing, and realizes the mining and diagnosis of fault information through the network [4]. At present, a trend of health monitoring is formed, which is based on online diagnosis and offline analysis and remote detection is supplemented. With the application of health monitoring technology, faults can be discovered as soon as pos- 
sible, and the reliability, safety and effectiveness of equipment can be improved, and the economic losses of company can be improved while minimizing the damage losses [5]. Therefore, it is very important to monitor and diagnose the state of mechanical equipment in real time.

At present, the widely used method at home and abroad is to install sensors on the workshop or equipment, and then use the technology of pattern recognition, machine learning and intelligent algorithms to integrate and analyze the sensor's data and obtain the final diagnosis results [6]. Due to limited function and measurement range, a single sensor can only reflect the property of the measured object from one side [7]. In order to obtain comprehensive equipment status, multi-sensor data need to be fused to extract key features so as to overcome the limitations of a single sensor, utilize multi-sensor information more effectively, reduce fuzziness and increase the accuracy of decision diagnosis [8].

There are many algorithms for multi-sensor data fusion, which include data-level fusion, feature-level fusion and decision-level fusion [9]. They have been widely applied in fault diagnosis, energy consumption analysis, target recognition and other fields [10]. Evidence theory is widely used in multi-sensor data fusion due to its advantages in handling uncertain information [11]. Evidence theory originates from solving multi-valued mapping problem. After the development of A.P. Dempster and G.Shafer, a set of "combined evidence" is developed to deal with the uncertainty reasoning [12]. Thus, evidence theory is also called DS theory. Compared with Bayesian probability theory, DS theory does not need to know the prior probability, and has the ability to directly express "uncertainty" and "do not know". Reference [13] proposed a diagnostic method of multi-sensor data fusion based on DS theory for uncertainty modeling. Reference [14] solved the problem of low accuracy and reliability in the diagnosis of single sensor by means of DS theory.

Although DS theory has unique advantage in data fusion, there is a fatal flaw. When the evidence is highly conflicting, the fusion result fails [15]. To solve this problem, a large number of research work has been carried out by scholars at home and abroad. Reference [16] assigned the probability of conflicting evidence to unknown set to solve the problem of strong conflict, but the result was not ideal when evidences were more than two pieces. Reference [17] introduced a credibility of evidence and assigned the probabilities of conflicting evidences to various propositions, but the synthesis result still differed from common sense. In Reference [18], the probability of evidence conflict is weighted according to the average support degree to each proposition, and a relatively perfect synthetic result was obtained, but the complete set was ignored. Reference [19] proposed a method of averaging combination of evidence, which converged quickly but ignored the connection between evidences. Reference [20] weighted and averaged the evidence before fusion, obtained stronger anti-interference ability, faster convergence rate. The above research methods either redistribute the basic probability assignment (BPA) or modify the fusion rule to reduce the degree of conflict. This paper presents a new fusion method to solve the problem of conflict, which not only adjusts the BPA of evidence but also modifies the fusion rule. The method is applied in monitoring and diagnosing the health status of rolling bearing and obtains a good diagnosis rate. 


\section{Preparation}

DS theory is an uncertain reasoning theory, which is first established in 1967 by Dempster [21] in his work on upper and lower probabilities and later developed in 1976 by his student, who is called Shafer [22]. DS theory not only emphasizes the objectivity of things, but also emphasizes the subjectivity of humans' estimation of things. Its greatest feature is the use of "interval estimation" for the description of uncertainty information. Compared to Bayesian networks, DS theory can produce a better fusion results by simple reasoning without knowing prior probability [23]. The basic mathematical concepts of DS theory are defined as follows.

Assume $\Omega=\left\{A_{1}, A_{2}, \ldots, A_{n}\right\}$ as a set, which $\Omega$ is a finite nonempty set of hypotheses, the set $\Omega$ is called recognition framework, which consists of $n$ mutually exclusive and exhaustive hypotheses $\mathrm{A}_{\mathrm{i}} \cdot 2^{\Omega}$ is a power set composed of all the subsets.

Definition 1 BPA is a primitive function $m$, which is a mapping from $2^{\Omega}$ to $[0,1]$, which should satisfy the following equation

$$
\left\{\begin{array}{c}
m(\Phi)=0 \\
\sum_{A \subseteq \Omega} m(A)=1
\end{array} .\right.
$$

$m(A)$ represents the initial support degree for proposition $A$. If $m(A)>0$, it is called a focal element.

Definition 2 The belief function is defined as

$$
\left\{\begin{array}{c}
B e l: 2^{\Omega} \rightarrow[0,1] \\
\operatorname{Bel}(A)=\sum_{B \subseteq A} m(B) \quad A \subseteq \Omega
\end{array} .\right.
$$

$\operatorname{Bel}(A)$ is called the belief function, also known as the lower limit function. It represents the complete trust of proposition $A$. It's easy to obtain the following equation from the definition 1

$$
\left\{\begin{array}{l}
\operatorname{Bel}(\Phi)=\mathrm{m}(\Phi)=0 \\
\operatorname{Bel}(\Omega)=\sum_{B \subseteq \Omega} m(B)
\end{array} .\right.
$$

Definition 3 The plausibility function is defined as

$$
\left\{\begin{array}{c}
\text { PI: } 2^{\Omega} \rightarrow[0,1] \\
P I(A)=1-\operatorname{Bel}(-\mathrm{A}) \quad A \subseteq \Omega
\end{array}\right.
$$

$P l(A)$ is called the plausibility function, also known as the upper limit function. It represents an uncertainty measure of proposition $A$.

Definition 4 Suppose that $m_{1}, m_{2}, \ldots, m_{n}$ are mutually independent BPA from $n$ different sensors in the same recognition framework. The DS combination rule can be defined as

$$
\left\{\begin{array}{c}
m(\Phi)=0 \quad A=\Phi \\
m(A)=(1-k)^{-1} \sum_{\cap A_{i=A}} \prod_{1 \leq i \leq n} m_{i}\left(A_{i}\right) \quad A \neq \Phi
\end{array}\right.
$$


Where $k=\sum_{\cap A_{i}=\Phi} \prod_{1 \leq i \leq n} m_{i}\left(A_{i}\right)$, it represents the degree of conflicts between evidences, which is called conflict factor.

Definition 5 The essence of DS theory is the orthogonal sum of evidences. It satisfies the following properties.

$$
\begin{gathered}
\text { Commutative law: } m_{1} \oplus m_{2}=m_{2} \oplus m_{1} \\
\text { Associate law: }\left(m_{1} \oplus m_{2}\right) \oplus m_{3}=m_{1} \oplus\left(m_{2} \oplus m_{3}\right)
\end{gathered}
$$

\section{Improvement}

In the traditional DS theory, conflict factor $\mathrm{k}$ represents the degree of conflicts between evidences. $0 \leq \mathrm{k} \leq 1$, $\mathrm{k}$ equals or approaches 0 , the fusion result is ideal; the bigger the $\mathrm{k}$ is, the fiercer the conflict between evidences is, and the more obvious the contradiction is. $(1-\mathrm{k})^{-1}$ is called a correction factor, and its role is to avoid assigning a non-zero probability to the empty set when the evidence is combined, and the confidence distribution discarded by the empty set is proportionally added to the nonempty set. However, this brings a great shortage of DS theory: it is impossible to deal with the evidence of high conflict. When $\mathrm{k}$ is 1 , the evidence is completely conflicting, DS theory cannot be used for fusion; when k approaches 1 , the evidence is high conflicting, and the result of the combination is often counter-intuitive [24]. The example in Table 1 illustrates this very well.

Table 1. Example of high and complete conflicts

\begin{tabular}{|c|c|c|c|c|c|c|c|}
\hline \multicolumn{4}{|c|}{ High Conflict } & \multicolumn{4}{c|}{ Complete Conflict } \\
\hline & $\boldsymbol{A}$ & $\boldsymbol{B}$ & $\boldsymbol{C}$ & & $\boldsymbol{A}$ & $\boldsymbol{B}$ & $\boldsymbol{C}$ \\
\hline $\mathrm{m}_{1}$ & 0.99 & 0.01 & 0 & $\mathrm{~m}_{1}$ & 1 & 0 & 0 \\
\hline $\mathrm{m}_{2}$ & 0 & 0.01 & 0.99 & $\mathrm{~m}_{2}$ & 0 & 1 & 0 \\
\hline $\mathrm{m}_{12}$ & 0 & 1 & 0 & $\mathrm{~m}_{12}$ & \multicolumn{3}{c|}{ Unable synthesis } \\
\hline $\mathrm{k}$ & \multicolumn{3}{|c|}{0.9999} & $\mathrm{k}$ & \multicolumn{3}{c|}{1} \\
\hline
\end{tabular}

In the case of high conflict, evidence $\mathrm{m}_{1}$ supports target $\mathrm{A}$ and evidence $\mathrm{m}_{2}$ supports target C. According to formula (5), the fusion result is target $\mathrm{B}$ and the conflict factor $\mathrm{k}$ is 0.9999 . This result is not consistent with common sense. This is the classic "Zadeh paradox". In the case of complete conflict, evidence $\mathrm{m}_{1}$ fully supports target A and evidence $\mathrm{m}_{2}$ fully supports target B. According to formula (5), the conflict factor $\mathrm{k}$ is 1 , causing the denominator of formula (5) to become 0 , which is not allowed mathematically. Under this circumstance, DS combination rule is unable to synthesis.

To solve this problem, an improved DS theory is proposed in this paper. First, a similarity matrix, which is calculated with the distance function, transforms into evidence weight. Second, sensor weight is introduced to distinguish data from different sensors. Then, we combines evidence and sensor weights to modify the BPA of evidence to reduce the conflicting. Finally, threshold $\lambda$ is introduced when the fusion rule 
is selected. The corresponding fusion formula is selected by comparing the relationship between $k$ and $\lambda[25]$.

\subsection{Evidence weight}

During the operation of equipment, data collected by sensors are not completely reliable. In order to obtain good diagnosis result, it is necessary to further verify the data consistency. The distance function is often utilized to test consistency. The smaller the distance is between two sensors, the higher the support is for each other and vice versa. Take two $n$-dimensional vectors $m_{1}\left(x_{11}, x_{12}, \ldots, x_{1 n}\right)$ and $m_{2}\left(x_{21}, x_{22}, \ldots, x_{2 n}\right)$ as an example. The distance between them is shown in Table 2 .

Table 2. Common distance formula

\begin{tabular}{|c|c|}
\hline Name & Formula \\
\hline Euclidean distance & $\mathrm{d}\left(m_{1}, m_{2}\right)=\sqrt{\sum_{k=1}^{n}\left(x_{1 k}-x_{2 k}\right)^{2}}$ \\
\hline Manhattan distance & $\mathrm{d}\left(m_{1}, m_{2}\right)=\sum_{k=1}^{n}\left|x_{1 k}-x_{2 k}\right|$ \\
\hline Chebyshev distance & $\mathrm{d}\left(m_{1}, m_{2}\right)=\max \left(\left|x_{1 k}-x_{2 k}\right|\right)$ \\
\hline Minkowski distance & $\mathrm{d}\left(m_{1}, m_{2}\right)=\sqrt[p]{\sum_{k=1}^{n}\left|x_{1 k}-x_{2 k}\right|^{p}}$ \\
\hline Mahalanobis distance & $\mathrm{d}\left(m_{1}, m_{2}\right)=\sqrt{\left(m_{1}-m_{2}\right)^{T} S^{-1}\left(m_{1}-m_{2}\right)}$ \\
\hline
\end{tabular}

Different distance functions have their own advantages and disadvantages. In practice, the appropriate distance function is selected according to needs. The Mahalanobis distance has nothing to do with the measurement and the inverse covariance matrix can get rid of the scale effects. It also can get rid of correlation jamming among variables. Therefore, we select the Mahalanobis distance to correct the evidence weight.

The Mahalanobis distance $\left(d_{i j}\right)$ between $m_{i}$ and $m_{j}$ can be calculated as

$$
d_{i j}\left(m_{i}, m_{j}\right)=\sqrt{\left(m_{i}-m_{j}\right)^{T} S^{-1}\left(m_{i}-m_{j}\right)} .
$$

where $T$ stands for transpose and $S$ stands for the covariance matrix. The distance matrix is defined as

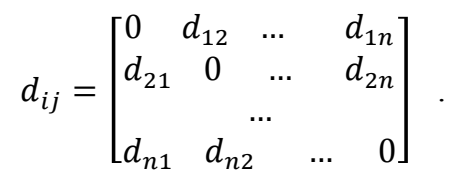

The similarity function $\left(S_{i j}\right)$ between $m_{i}$ and $m_{j}$ can be defined as 


$$
S_{i j}=\sqrt{2}-d_{i j} .
$$

It is obvious that the similarity and distance function are inverse relations. The similarity matrix is defined in the same way as shown below

$$
s_{i j}=\left[\begin{array}{cccr}
1 & s_{12} & \ldots & s_{1 n} \\
s_{21} & 1 & \ldots & s_{2 n} \\
& & \ldots & \\
s_{n 1} & s_{n 2} & \ldots & 1
\end{array}\right] .
$$

The support measurement (Sup $\left.\left(m_{i}\right)\right)$ and credibility $\left(\right.$ Cred $\left.\left(m_{i}\right)\right)$ of evidence $m_{i}$ can be introduced separately as

$$
\begin{gathered}
\operatorname{Sup}\left(m_{i}\right)=\sum_{j=1, j \neq i}^{n} S_{i j}\left(m_{i}, m_{j}\right) . \\
\operatorname{Cred}\left(m_{i}\right)=\operatorname{Sup}\left(m_{i}\right) / \sum_{i=1}^{n} \operatorname{Sup}\left(m_{i}\right) .
\end{gathered}
$$

It is clear that the Cred $\left(m_{i}\right)$ will increase with increases of $\mathrm{S}_{\mathrm{ij}}$. So we select the Cred $\left(m_{i}\right)$ as evidence weight.

\subsection{Sensor weight}

Influenced by the precision and environment, the sensor cannot fully reflect the true state of the measured object, thus affecting the fusion precision. According to experience and expert system, sensor weight $\omega\left(s_{i}\right)$ can represent the dominance and importance of prior knowledge in actual application. It should satisfy $\omega\left(s_{i}\right) \in[0,1]$ and $\sum \omega\left(s_{i}\right)=1$.

There are many different ways of setting sensor weights. Delphi method is one of the widely used. The Delphi method is also called the expert survey method. The organizer designs the questionnaire and sends it to experts in an anonymous manner according to the prescribed process [26]. Through multiple interactions between experts and questionnaires, a basically consistent view is finally reached as a result of the prediction. The advantages of this method are simple, scientific and practical. It can give full play to the role of experts and obtain reliable conclusions [27]. The simplified Delphi method is used in this paper. According to the sensor properties and installation location, experimental environment and workbench, a corresponding questionnaire is designed and distributed to front-line workers in the workshop. The worker then completes the questionnaire based on their prior knowledge. Finally, the average results of the questionnaire are used as the sensor weights.

In Section 4, the data from different sensors are taken as evidence. Three accelerometer sensors are installed in the bearing seat which located at $\mathrm{X}, \mathrm{Y}$ and $\mathrm{Z}$ axis to collect vibration signals in different states. According to the different location, combined with the prior knowledge, different weights are given to the sensor $\omega\left(\mathrm{s}_{1}\right)=0.5, \omega\left(\mathrm{s}_{2}\right)=0.4, \omega\left(\mathrm{s}_{3}\right)=0.1$. 


\subsection{BPA Correction}

The traditional DS theory leads to the instability and unreliability of the fusion result because of the high conflict factor. Therefore, the new BPA of evidence is modified by means of evidence weight Cred $\left(m_{i}\right)$ and sensor weight $\omega\left(e_{i}\right)$. To ensure the normalization of fusion results, the new BPA should be normalized before combination, which is defined as follows:

$$
\begin{gathered}
m_{i}^{*}(A)=\sum_{i=1}^{n} m_{i}(A) * \operatorname{Cred}\left(m_{i}\right) * \omega\left(s_{i}\right) \\
m_{i}^{\#}(A)=m_{i}^{*}(A) / \sum_{A \subseteq \Omega} m_{i}^{*}(A)
\end{gathered}
$$

\subsection{Improvement of the combination rule}

In the application of DS theory, when $\mathrm{k}$ is 1 , the evidence is full conflicting; when $\mathrm{k}$ is 0 , the evidence is compatible. When $\mathrm{k}$ approaches 1 , it means strong conflict, on the contrary, it is weak conflict. Traditional DS theory is selected when evidence is compatible or weak conflict, and the improved rule is adopted when the evidence is in full or strong conflict [25]. The improved method not only retains the advantages of DS theory, but also reduces the impact of conflicting on the synthesis results.

$$
\begin{aligned}
& \mathrm{k}=\sum_{\cap A_{i}=\varnothing} \prod_{1 \leq i \leq n} m_{i}^{\#}\left(A_{i}\right) \\
& \mathrm{q}(\mathrm{A})=\frac{1}{n} \sum_{i=1}^{n} m_{i}^{\#}\left(A_{i}\right) \\
& \left\{\begin{array}{lr}
m(\emptyset)=0 & A=\phi \\
m(A)= \begin{cases}\frac{1}{1-k} \sum_{\cap A_{i}=A} \prod_{1 \leq i \leq n} m_{i}^{\#}\left(A_{i}\right) & k<\lambda \\
\sum_{\cap A_{i=A}} \prod_{1 \leq i \leq n} m_{i}^{\#}\left(A_{i}\right)+k * q(A) & k>\lambda\end{cases} & A \neq \phi \text { and } \mathrm{A} \neq \mathrm{U} \\
m(U)=1-\sum_{i=1}^{n} m_{i}^{\#}\left(A_{i}\right) & A=\mathrm{U}
\end{array}\right.
\end{aligned}
$$

Here, $\phi$ is an empty set, and $U$ is a full set.

\subsection{Effectiveness analysis}

In order to illustrate the effectiveness of the improved method, we compares the fusion result of different methods. Four sensors identify three targets [20]. The BPA

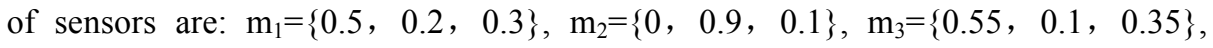
$\mathrm{m}_{4}=\{0.55,0.1,0.35\}$. The comparison are shown in Table 3 .

According to Table 3 , the evidence $\mathrm{m}_{2}$ denies the target $A$. No matter how many pieces of evidence support $A$, the fusion result cannot been changed. It indicates that the traditional DS theory can't handle conflict effectively, and there is a "one vote veto" problem. The result of Reference [16] shows that the uncertainty of result is given to an unknown set $U$. As the number of evidence increase, the uncertainty increase. In Reference [17], it eliminates the phenomenon of "one vote veto". Howev- 
er, the proportion of unknown set $U$ is still large and the decision cannot be decided. Reference [18] simplifies the fusion rule on the basis of Reference [17], and it identifies the target $A$ accurately when evidences are added to four pieces. Reference [20] introduces the credibility of evidence and correctly identifies the target $A$ when the evidence is added to three pieces. The improved method adjusts the BPA and combination rules, so that the correct result can be quickly identified under two pieces of evidence.

Table 3. Fusion results with different methods

\begin{tabular}{|c|c|c|c|}
\hline Method & $\mathbf{m} 1 \oplus \mathbf{m} 2$ & $\mathrm{~m} 1 \oplus \mathrm{m} 2 \oplus \mathrm{m} 3$ & $\mathbf{m} 1{ }^{\oplus} \mathbf{m} 2 \mathbf{2}^{\oplus} \mathbf{m} 3^{\oplus} \mathbf{m} 4$ \\
\hline \multirow{3}{*}{ Traditional DS } & $\mathrm{m}(\mathrm{A})=0$ & $\mathrm{~m}(\mathrm{~A})=0$ & $\mathrm{~m}(\mathrm{~A})=0$ \\
\hline & $m(B)=0.8571$ & $m(B)=0.6316$ & $\mathrm{~m}(\mathrm{~B})=0.3288$ \\
\hline & $\mathrm{m}(\mathrm{C})=0.1429$ & $\mathrm{~m}(\mathrm{C})=0.3684$ & $m(C)=0.6712$ \\
\hline \multirow{4}{*}{ Reference[16] } & $\mathrm{m}(\mathrm{A})=0$ & $\mathrm{~m}(\mathrm{~A})=0$ & $\mathrm{~m}(\mathrm{~A})=0$ \\
\hline & $\mathrm{m}(\mathrm{B})=0.1800$ & $\mathrm{~m}(\mathrm{~B})=0.0180$ & $\mathrm{~m}(\mathrm{~B})=0.0018$ \\
\hline & $\mathrm{m}(\mathrm{C})=0.0300$ & $\mathrm{~m}(\mathrm{C})=0.0105$ & $\mathrm{~m}(\mathrm{C})=0.0037$ \\
\hline & $m(U)=0.7900$ & $m(U)=0.9715$ & $m(U)=0.9945$ \\
\hline \multirow{4}{*}{ Reference[17] } & $\mathrm{m}(\mathrm{A})=0.0896$ & $\mathrm{~m}(\mathrm{~A})=0.1598$ & $\mathrm{~m}(\mathrm{~A})=0.1941$ \\
\hline & $\mathrm{m}(\mathrm{B})=0.3772$ & $\mathrm{~m}(\mathrm{~B})=0.2006$ & $\mathrm{~m}(\mathrm{~B})=0.1595$ \\
\hline & $\mathrm{m}(\mathrm{C})=0.1017$ & $\mathrm{~m}(\mathrm{C})=0.1247$ & $\mathrm{~m}(\mathrm{C})=0.1371$ \\
\hline & $m(U)=0.4315$ & $m(U)=0.5149$ & $m(U)=0.5092$ \\
\hline \multirow{4}{*}{ Reference[18] } & $\mathrm{m}(\mathrm{A})=0.1975$ & $\mathrm{~m}(\mathrm{~A})=0.3400$ & $m(A)=0.3978$ \\
\hline & $m(B)=0.6145$ & $m(B)=0.4066$ & $\mathrm{~m}(\mathrm{~B})=0.3250$ \\
\hline & $\mathrm{m}(\mathrm{C})=0.1880$ & $\mathrm{~m}(\mathrm{C})=0.2534$ & $\mathrm{~m}(\mathrm{C})=0.2772$ \\
\hline & $\mathrm{m}(\mathrm{U})=0$ & $\mathrm{~m}(\mathrm{U})=0$ & $\mathrm{~m}(\mathrm{U})=0$ \\
\hline \multirow{3}{*}{ Reference[20] } & $\mathrm{m}(\mathrm{A})=0.1543$ & $m(A)=0.5816$ & $m(A)=0.8060$ \\
\hline & $m(B)=0.7469$ & $\mathrm{~m}(\mathrm{~B})=0.2439$ & $\mathrm{~m}(\mathrm{~B})=0.0482$ \\
\hline & $\mathrm{m}(\mathrm{C})=0.0988$ & $\mathrm{~m}(\mathrm{C})=0.1745$ & $\mathrm{~m}(\mathrm{C})=0.1458$ \\
\hline \multirow{4}{*}{ Improved DS } & $m(A)=0.7469$ & $m(A)=0.8358$ & $m(A)=0.9299$ \\
\hline & $\mathrm{m}(\mathrm{B})=0.1543$ & $\mathrm{~m}(\mathrm{~B})=0.1097$ & $\mathrm{~m}(\mathrm{~B})=0.0491$ \\
\hline & $\mathrm{m}(\mathrm{C})=0.0988$ & $\mathrm{~m}(\mathrm{C})=0.0545$ & $\mathrm{~m}(\mathrm{C})=0.0210$ \\
\hline & $\mathrm{m}(\mathrm{U})=0$ & $\mathrm{~m}(\mathrm{U})=0$ & $\mathrm{~m}(\mathrm{U})=0$ \\
\hline
\end{tabular}

\subsection{Robustness analysis}

Robustness is used to measure the fault tolerance and portability of the model. For the method in this article, the BPA of evidence is easily interfered by external factors that affects the final synthesis result. As can be seen from Table 3, the traditional DS theory is more sensitive to the BPA of evidence. There is a problem of "one vote veto" and poor robustness. In Reference [16], the uncertainty of result is given to an unknown set $U$. Reference [17] eliminates the "one vote veto" phenomenon, but it still 
cannot make a decision. The robustness of method in Reference [18] has been enhanced and the correct result can be obtained with four pieces of evidence. In Reference [20], the robustness is further strengthened and the correct result can be obtained with three pieces of evidence. The improved method can achieve the fusion result with only two pieces of evidence, which has better robustness than the previous methods.

\section{Application}

\subsection{Monitoring and diagnosis of health status of rolling bearing}

In order to illustrate the practicability, the improved algorithm is applied in the assessment of the health status of rolling bearing. Rolling bearing is an important part of rotary instrument, which is usually composed of four parts: inner ring, outer ring, ball and cage. Failure of any part will cause a potential safety accident [28]. We take the SKF 6205-2RS bearing as the research object. The fault grooves with a width of 0.4 $\mathrm{mm}$ and a depth of $0.2 \mathrm{~mm}$ are manufactured by spark erosion technique. Five conditions are simulated respectively: Health $(\mathrm{H})$, inner race fault $(\mathrm{I})$, ball fault $(\mathrm{B})$, outer race fault $(\mathrm{O})$ and cage fault $(\mathrm{C})$. Three accelerometers are installed on the bearing seat which located at $\mathrm{X}, \mathrm{Y}$ and $\mathrm{Z}$ axis to collect 100 sets vibration signals in different states separately. The speed is $1797 \mathrm{rpm}$, sampling frequency is $24 \mathrm{kHz}$ and sample length is 2048 for all conditions of the bearing. In different failure modes, the output of sensors are first amplified and then sent to analog/digital (A/D) converter and stored in a computer. The signal is de-noised by the $3 \sigma$ criterion. The statistical features are extracted by fast Fourier transform (FFT). To avoid contingency, 70 sets data are randomly selected as training set, and the remaining is taken as testing set. Both of them are sent into the classifier to distinguish fault probability. The fault probability is taken as the BPA of evidence. Finally, the improved DS theory is used to make the fusion decision to obtain the bearing fault category. The schematic diagram of algorithm is shown in Fig. 1.

\subsection{Simulation analysis}

We assume that the recognition framework is $\Omega=\{H, I, B, O, C\}$, where $H, I, B, O$ and $C$ separately represent health, inner race fault, ball fault, outer race fault and cage fault. Because the output of the acceleration sensor is an analog signal, it cannot be used directly as the BPA of evidence. It needs to be transformed into digital signal by means of signal processing unit, and then transformed into probability by neural network classifier to be the BPA of evidence. The genetic algorithm (GA) is adopted to optimize the initial weights and thresholds of back propagation (BP) neural network to avoid the local optimization [29]. This model is called GA-BP. The flow chart of GA-BP is shown in Fig. 2.

The BP network parameters are set as follows:

Maximum number of training steps: 1000 


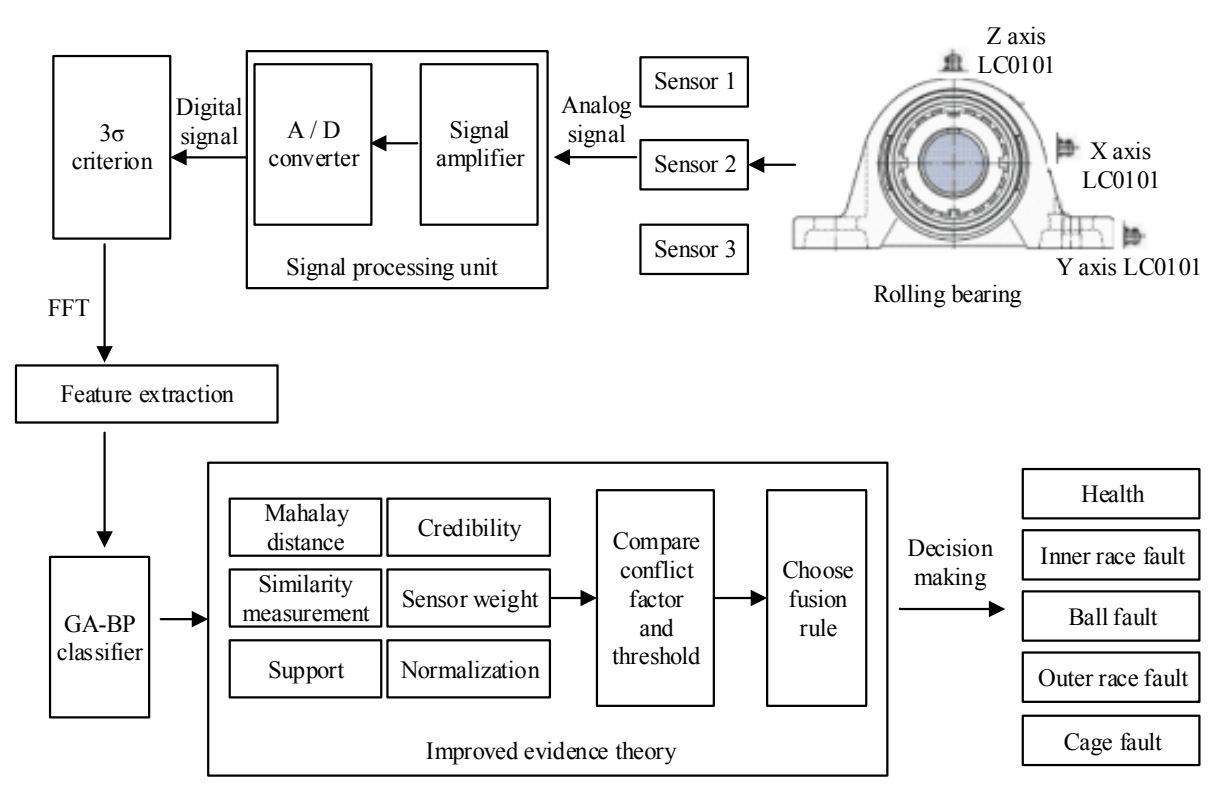

Fig. 1. Schematic diagram of the algorithm

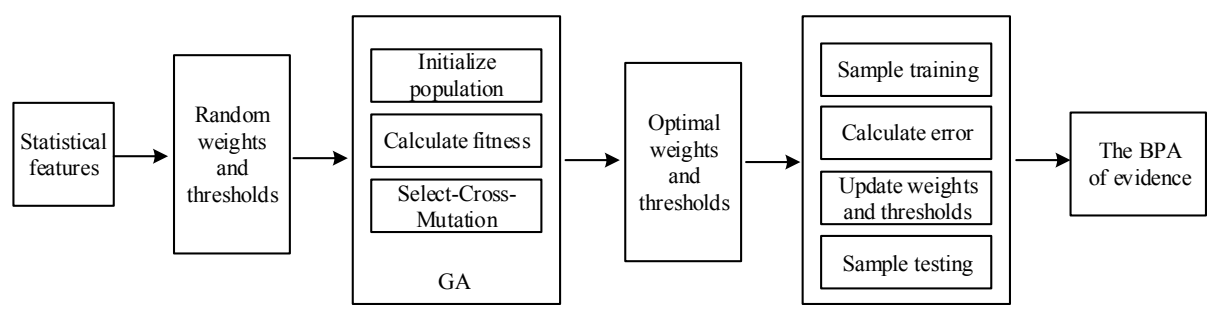

Fig. 2. Flow chart of GA-BP

Minimum Target Training Error: 0.1

Learning rate: 0.1

The number of intervals displaying result: 10

The GA parameters are set as follows:

The size of population: 30

The number of evolutions: 200

The crossover probability: 0.4

The mutation probability: 0.1

The fusion result is compared with the median Voting Fusion [30], the proportional conflict redistribution 5 (PCR5) fusion [31] and the traditional DS theory. To facilitate the analysis of results, only two sets of sample data corresponding to each sensor are extracted, and the results are shown in Table 4. 
Table 4. Result of different fusion methods

\begin{tabular}{|l|c|c|c|c|c|c|c|}
\hline & \multicolumn{4}{|c|}{ BPA } & \multicolumn{2}{c|}{ Result } \\
\hline \multicolumn{1}{|c|}{ No. } & $\boldsymbol{H}$ & $\boldsymbol{I}$ & $\boldsymbol{B}$ & $\boldsymbol{O}$ & $\boldsymbol{C}$ & $\begin{array}{c}\text { Diagno- } \\
\text { sis }\end{array}$ & Actual \\
\hline Sensor-1-1 & 0.0582 & 0.3653 & 0.3513 & 0.0368 & 0.1884 & $\mathrm{I}$ & $\mathrm{B}$ \\
\hline Sensor-1-2 & 0.0034 & 0.0177 & 0.4513 & 0.1090 & 0.4186 & $\mathrm{~B}$ & $\mathrm{~B}$ \\
\hline Sensor-2-1 & 0.1527 & 0.4060 & 0.1785 & 0.0525 & 0.2103 & $\mathrm{I}$ & $\mathrm{B}$ \\
\hline Sensor-2-2 & 0.0045 & 0.3643 & 0.5947 & 0.0059 & 0.0306 & $\mathrm{~B}$ & $\mathrm{~B}$ \\
\hline Sensor-3-1 & 0.1438 & 0.0184 & 0.2032 & 0.4321 & 0.2025 & $\mathrm{O}$ & $\mathrm{B}$ \\
\hline Sensor-3-2 & 0.4767 & 0.0338 & 0.2312 & 0.1765 & 0.0818 & $\mathrm{H}$ & $\mathrm{B}$ \\
\hline Median voting & 0.3331 & 0.1216 & 0.5947 & 0.2222 & 0.2358 & $\mathrm{~B}$ & $\mathrm{~B}$ \\
\hline PCR5 fusion & 0.0144 & 0.0157 & 0.9214 & 0.0424 & 0.0128 & $\mathrm{~B}$ & $\mathrm{~B}$ \\
\hline Traditional DS & 0.0041 & 0.0538 & 0.9074 & 0.0034 & 0.0313 & $\mathrm{~B}$ & $\mathrm{~B}$ \\
\hline Improved DS & 0.0038 & 0.0160 & 0.9647 & 0.0033 & 0.0122 & $\mathrm{~B}$ & $\mathrm{~B}$ \\
\hline
\end{tabular}

Table 4 shows that different sensors make different diagnostic results. The diagnostic result of Sensor-1-1 is wrong, but there is only 0.014 difference between the diagnosis of the inner race fault $(0.3653)$ and the actual situation of the ball fault (0.3513). The diagnosis result of Sensor-1-2 is right, but the ball fault (0.4513) is very close to the cage failure (0.4186), which indicates that Sensor-1 is uncertainty and the results are not completely reliable. Two sets data of Sensor-2, one of them is accurate and the other one is wrong, but the accuracy is only 0.4060. Two sets data of Sensor-3 are diagnosed incorrectly. The probability is less than 0.5 and the result is unreliable.

Figure 3 shows that the single sensor cannot accurately and comprehensively reflect the healthy state of rolling bearing due to its limitation. It is necessary to combine the data from multi-sensors to obtain an explanation and description of consistency of measured object. The bearing fault can be quickly and accurately decided by DS theory to fuse multi-sensor data. The traditional DS theory achieves the probability of 0.9074 , while the improved DS is up to 0.9647 , which is obviously different from others. The reliability and necessity of the fusion are further verified.

From the results of Table 4, all four methods make correct judgment on the healthy status of rolling bearing. However, comparison of results in Figure 4, the improved DS algorithm obtains the highest diagnostic rate $(0.9647)$, followed by PCR5 fusion (0.9214), the third with the traditional DS theory (0.9074), the median voting fusion lowest (0.5947). Because the improved algorithm replaces the BPA of evidence with a new BPA which corrected by evidence and sensor weights. The fusion rule is selected according to the relationship between $k$ and $\lambda$, so that the evidence with high confidence level is higher and higher, the evidence with low confidence level is getting lower and lower. Finally, we get the ideal diagnosis rate. The PCR5 method focuses on the full conflict of evidence. It allocates the probability according to the original confidence level, which is relatively conservative and obtains the second highest diagnosis rate. The diagnosis rate of the traditional DS theory is higher than that of any single sensor, which fully embodies the advantages of fusion. The essence 
of median voting fusion is "Voting by voting, most of pass". It is a simple, intuitive and fast method without complex operation. It can be completed in the shortest time $\mathrm{O}(\mathrm{N})$, but the diagnostic rate is the lowest among four methods.

Table 5 shows that the diagnostic rate of all four fusion methods is higher than that of the single sensor. The diagnostic rate of the testing set is lower than that of the training set. It is consistent with the actual. The time complexity of traditional DS theory is $\mathrm{O}\left(\mathrm{N}^{3}\right)$ and that of the improved DS algorithm is $\mathrm{O}\left(\mathrm{N}^{2}\right)$. Thus, the improved method not only reduces the system time-consuming $\left(\mathrm{N}^{3}\right.$ to $\left.\mathrm{N}^{2}\right)$, but also increases the diagnostic rate to 0.9647 . Therefore, the improvement of DS theory in this paper is effective and practical.

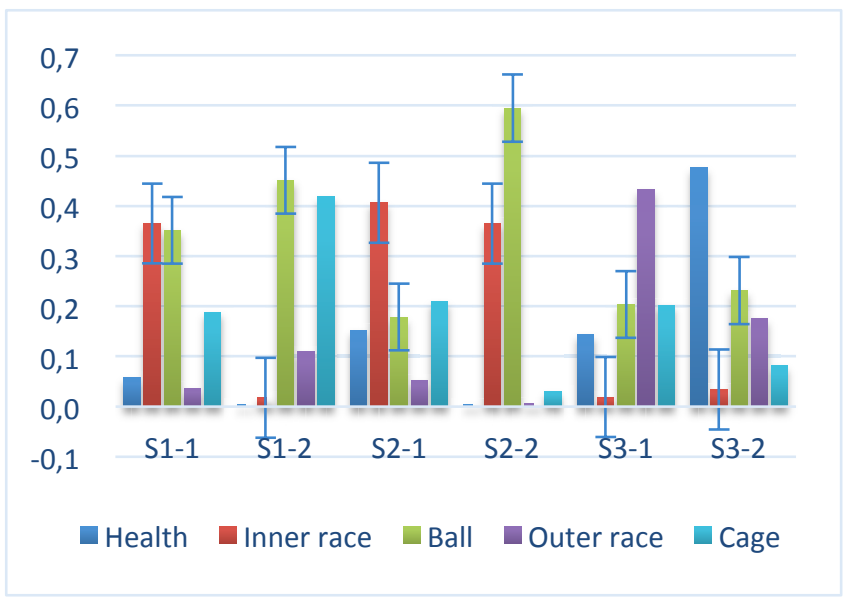

Fig. 3. Fault diagnosis result of single sensor

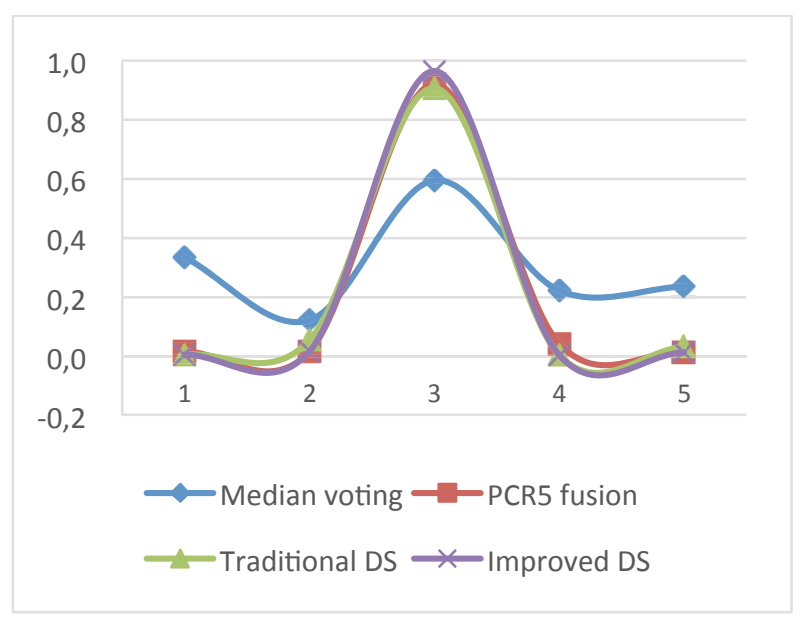

Fig. 4. Comparison of four diagnostic results 
Table 5. Diagnostic results of single and multi-sensors

\begin{tabular}{|l|c|c|c|}
\hline \multirow{2}{*}{ Method } & \multicolumn{2}{|c|}{ Diagnostic rate (\%) } & \multirow{2}{*}{ Time complexity } \\
\cline { 2 - 3 } & Training set & Testing set & \\
\hline Sensor-1 & 75.5 & 72.3 & $\mathrm{O}(1)$ \\
\hline Sensor-2 & 78.9 & 75.2 & $\mathrm{O}(1)$ \\
\hline Sensor-3 & 59.1 & 55.7 & $\mathrm{O}(1)$ \\
\hline Median voting fusion & 81.2 & 78.3 & $\mathrm{O}(\mathrm{N})$ \\
\hline PCR5 fusion & 92.6 & 90.0 & $\mathrm{O}\left(\mathrm{N}^{2}\right)$ \\
\hline Traditional DS fusion & 90.0 & 88.6 & $\mathrm{O}\left(\mathrm{N}^{3}\right)$ \\
\hline Improved DS fusion & 96.8 & 94.7 & $\mathrm{O}\left(\mathrm{N}^{2}\right)$ \\
\hline
\end{tabular}

\section{Conclusion}

The monitoring and diagnosis of the healthy status of mechanical equipment greatly improves the production efficiency, reduces the maintenance cost, prolongs the life of equipment and helps the national economy to develop more rapidly. In order to monitor the health status of equipment, an improved DS theory is proposed in this paper. The BPA of evidence is adjusted by evidence and sensor weights, and then select the fusion rule based on the relationship between $k$ and $\lambda$ to carry out multisensor data fusion. The effectiveness and practicability of method are verified by the diagnosis of the health state of rolling bearing. The robustness analysis of algorithm further clarifies that the improved method not only inherits the advantages of traditional DS theory in dealing with uncertain information, but also solves the problem of fusion failure of evidence conflict. By comparing the diagnostic results of multiple and single sensors, it shows that the decision-making of multi-sensor can effectively improve the accuracy and reliability of mechanical equipment fault diagnosis. To a certain extent, the requirements for the performance of a single sensor are reduced. It has some practical significance in monitoring the health status of mechanical equipment.

\section{Acknowledgment}

This work is supported by National Natural Science Foundation of China (51475097), National Science Foundation of China under Grant (61640209), Ministry of Industry and Intelligent Manufacturing Demonstration Project (Ministry of Industry [2016]213) and Key Basic Research Program of Guizhou Province of China (No. [2014]2001). 


\section{$7 \quad$ References}

[1] Wang, X. (2014). Research on Remote Condition Monitoring System in Coal Mine. International Journal of Online Engineering. 10(1). pp.37. https://doi.org/10.3991/ijoe.v10i1. 3197

[2] Lee, J., Wu, F., Zhao, W., Ghaffari, M., Liao, L. and Siegel, D. (2014). Prognostics and health management design for rotary machinery systems - Reviews, methodology and applications. Mechanical Systems \& Signal Processing. 42(1-2). pp.314-334. https://doi.org/10.1016/j.ymssp.2013.06.004

[3] Ming, Y., Dong, C. and Xu, D. (2016). Review of Gear Fault Diagnosis Methods Based on Motor Drive System. Transactions of China Electrotechnical Society. 31(19). pp.132-140.

[4] Xing, W.U., Mao, J.L. and Chi, Y.L. (2009). Conceptual Model for Equipment Condition Monitoring and Diagnosis System. Computer Engineering. 35(12). pp.229-232.

[5] Wen, C.L., Fei-Ya, L.V., Bao, Z.J., Liu, M.Q., Automation, S.O. and University, H.D. (2016). A Review of Data Driven-based Incipient Fault Diagnosis. Acta Automatica Sinica. 42(9). pp.1285-1299.

[6] Cao, H., Fan, F., Zhou, K. and He, Z. (2016). Wheel-bearing Fault Diagnosis of Trains using Empirical Wavelet Transform. Measurement. 82(pp.439-449. https://doi.org/10.1016/ j.measurement.2016.01.023

[7] Xiao, F. (2017). A Novel Evidence Theory and Fuzzy Preference Approach-Based MultiSensor Data Fusion Technique for Fault Diagnosis. Sensors. 17(11). pp.2504. https://doi.org/10.3390/s17112504

[8] Lin, L. (2016). Multi-sensor Information Fusion Method Based on BP Neural Network. International Journal of Online Engineering. 12(5). pp.53.

[9] Wen, Y., Tan, J.W., Zhan, H. and Sun, X.B. (2016). Fault Diagnosis Based on Multisensor Data Fusion for Numerical Control Machine. International Journal of Online Engineering. 12(2). pp.29. https://doi.org/10.3991/ijoe.v12i02.5040

[10] Zhao, C., Tang, C.R. and Wan, S. (2011). Multisensor Information Fusion Based on D-S Evidence Theory and BP Neural Network. Automation \& Instrumentation. 567(pp.113117.

[11] Wang, X. and Song, Y. (2017). Uncertainty measure in evidence theory with its applications. Applied Intelligence. 6). pp.1-17. https://doi.org/10.1007/s10489-017-1024-y

[12] Tang, Y., Zhou, D., Xu, S. and He, Z. (2017). A Weighted Belief Entropy-Based Uncertainty Measure for Multi-Sensor Data Fusion. Sensors. 17(4).

[13] Jiang, W., Hu, W. and Xie, C. (2017). A New Engine Fault Diagnosis Method Based on Multi-Sensor Data Fusion. Applied Sciences. 7(3). pp.280. https://doi.org/10.3390/app 7030280

[14] Ma, B., Hao, L.C., Zhang, W.J., Dai, J. and Han, Z.H. (2012). Research on Equipment Fault Diagnosis Method Based on Multi-Sensor Data Fusion. Advanced Materials Research. 466-467(pp.1222-1226. https://doi.org/10.4028/www.scientific.net/AMR.466467.1222

[15] Jiang, W. and Zhan, J. (2017). A modified combination rule in generalized evidence theory. Applied Intelligence. 46(pp.1-11. https://doi.org/10.1007/s10489-016-0851-6

[16] Yager R R. (1987). On the Dempster-Shafer Framework and New Combination Rules. Information Sciences. 41(2). pp.93-137. https://doi.org/10.1016/0020-0255(87)90007-7

[17] Sun, Q., Ye, X. and Gu, W. (2000). A New Combination Rules of Evidence Theory. Acta Electronica Sinica. 28(8). pp.117-119.

[18] Li, B., Wang, B. Wei, J., Qian, C. and Huang, Y. (2002) An Efficient Combination Rule of Evidence Theory. Journal of Data Acquisition and Processing. 17(1). pp.33-36. 
[19] Murphy, C.K. (2000). Combining belief functions when evidence conflicts. Decision Support Systems. 29(1). pp.1-9. https://doi.org/10.1016/S0167-9236(99)00084-6

[20] Deng, Y., Shi, W. and Zhu, Z. (2004). Efficient Combination Approach of Conflict Evidence. J . Inf rared Millim. Waves. 23(1). pp.27-32.

[21] Dempster, A.P. (1967). Upper and Lower Probabilities Induced by a Multivalued Mapping. Annals of Mathematical Statistics. 38(2). pp.325-339. https://doi.org/10.1214/aoms/ 1177698950

[22] Shafer, G. (1976). A Mathematical Theory of Evidence. Princeton University Press, Princeton, New Jersey, USA.

[23] Li, Y., Chen, J., Ye, F. and Liu, D. (2015). The Improvement of DS Evidence Theory and Its Application in IR/MMW Target Recognition. Journal of Sensors,2016,(2015-12-28). 2016(6). pp.1-15.

[24] Ye, F., Chen, J. and Li, Y. (2017). Improvement of DS evidence theory for multi-sensor conflicting information.

[25] Yao, X.M., Li, S.B. and Zhang, A.S. (2018). Equipment Condition Monitoring and Diagnosis System Based on Evidence Weight. International Journal of Online Engineering. 14(2). pp.143-154. https://doi.org/10.3991/ijoe.v14i02.7731

[26] Linstone, H.A. and Turoff, M. (1976). The Delphi method : Techniques and applications. Journal of Marketing Research. 18(3). pp.363-364.

[27] Okoli, C. and Pawlowski, S.D. (2005). The Delphi method as a research tool: an example, design considerations and applications. Information \& Management. 42(1). pp.15-29. https://doi.org/10.1016/j.im.2003.11.002

[28] Yao, X., Li, S. and Hu, J. (2017). Improving Rolling Bearing Fault Diagnosis by DS Evidence Theory Based Fusion Model. Journal of Sensors,2017,(2017-10-22). 2017(1). pp.114.

[29] Feng, Z., Shi, W. and Hu, R. (2013). A Method for Multi-Sensor Information Fusion Based on Combining GA-BP Network with D-S Evidence Theory. International Journal of Digital Content Technology \& Its Applications. 7(8). pp.75-83. https://doi.org/10.4156/ jdcta.vol7.issue8.9

[30] Zhou, G., Ge, S. and Yang, L. (2010). Fault Diagnosis Method for Nuclear Power Plants Based on Neural Networks and Voting Fusion. Atomic Energy Science and Technology. 44(1). pp.367-372

[31] Zhai, X., Hu, J., Xie, S., Li, J. and Li, Q. (2012). Diagnosis of Aero-engine with Early Vibration Fault Symptom using DSmT. Journal of Aerospace Power. 27(2). pp.301-306.

\section{Authors}

Xuemei Yao is now pursuing her doctor degree in the Key Laboratory of Advanced Manufacturing Technology, Ministry of Education at Guizhou University, Guiyang, China, 550025. Her research interests include Machine Learning and computational intelligence (yaomei0119@126.com).

Shaobo Li is currently a professor of School of Mechanical Engineer at Guizhou University, Guiyang, China, 550025. His research interests include computational intelligence, manufacturing information system and interest things of technology. He is a member of the IEEE, CMES and CCF (lishaobo@gzu.edu.cn). 
Yong Yao is now pursuing his master degree in the School of Mechanical Engineer at Guizhou University, Guiyang, China, 550025. His research interests include mechanical manufacturing and automation(773240630@qq.com).

Xiaoting Xie is now pursuing his master degree in the Key Laboratory of Advanced Manufacturing Technology, Ministry of Education at Guizhou University, Guiyang, China, 550025. Her research interests include mechanical engineering (1252778652@qq.com).

Article submitted 28 January 2018. Resubmitted 18 March and 24 March 2018. Final acceptance 29 March 2018. Final version published as submittedby the authorts. 